\title{
Palliative radiotherapy to dominant symptomatic lesion in patients with hormone refractory prostate cancer (PRADO)
}

Jesper Carl ${ }^{1}$, Dirk Rades ${ }^{2 *}$, Claudia Doemer ${ }^{2}$, Cornelia Setter ${ }^{3}$, Jürgen Dunst ${ }^{3}$ and Niels Henrik Holländer ${ }^{1}$

\begin{abstract}
Background: This study was conducted to investigate a new short-course radiotherapy regimen for patients with metastatic hormone refractory prostate cancer (HRPC) presenting with a dominant debilitating symptom.

Methods / design: This is an international, multi-center single arm prospective feasibility study that aims to include 34 patients with HRPC and a dominant debilitating symptom. The dominant symptomatic lesion will receive $4 \times 5$ Gy of high-precision radiotherapy, and the most aggressive part of the lesion $4 \times 7$ Gy using a simultaneous integrated boost technique. Based on advanced magnetic resonance imaging (MRI), an apparent diffusion coefficient (ADC) map will be calculated for the lesion using diffusion weighted imaging sequences. The dominant symptomatic lesion (GTV1) is drawn manually using the information from T2W-MRI and computed tomography scans. The most aggressive part of the dominant lesion (GTV2) is defined by using the ADC map. An auxiliary volume is created including only voxels in the GTV1 that presents with ADC values below $1200 \times 10^{-6} \mathrm{~mm}^{2} / \mathrm{s}$. The most aggressive part is defined as voxels with an ADC value below the median ADC value. Primary endpoint is feasibility, i.e. proportion of patients who complete radiotherapy with $\geq 90 \%$ of the prescribed dose. Secondary endpoints include dominant symptom score, progression-free survival (freedom from symptoms), overall survival, acute toxicity, quality of life, change in ADC from baseline to end of treatment and 6 months following treatment.
\end{abstract}

Discussion: If this new radiotherapy regimen proves to be feasible, a prospective randomized phase II/III dose escalation study will be designed in order to improve the outcomes of palliative radiotherapy of symptomatic metastatic HRPC.

Study status: The study is ongoing and will be recruiting patients soon.

Trial registration: clinicaltrials.gov NCT03658434. Initially registered on 30th of July, 2018

Keywords: Prostate cancer, Metastatic disease, Dominant symptomatic lesion, High-precision radiotherapy, Hypofractionation, Feasibility

\footnotetext{
* Correspondence: rades.dirk@gmx.net

${ }^{2}$ Department of Radiation Oncology, University of Lübeck, Lübeck, Germany

Full list of author information is available at the end of the article
}

(c) The Author(s). 2019 Open Access This article is distributed under the terms of the Creative Commons Attribution 4.0 International License (http://creativecommons.org/licenses/by/4.0/), which permits unrestricted use, distribution, and 


\section{Background and rationale}

Prostate cancer is the major cause of cancer death in men living in developed countries. Large autopsy studies found that $35 \%$ of the patients with prostate cancer had metastatic disease. The most common metastatic sites include bone (90\%), lung (46\%), liver (25\%) and pleura (21\%) [1]. Patients may also present with disabling symptoms from local pelvic disease progression with an incidence of 10$18 \%$ or higher in node-positive patients treated with antiandrogen therapy alone [2-4]. Pelvic radiotherapy (RT) can provide effective palliation by contributing to relief of hematuria, pain and other symptoms [5-9].

Metastatic or locally advanced prostate cancer (PC) initially responds well to hormonal manipulation by androgen withdrawal and peripheral androgen blockade. However, such patients have high risk of developing a hormone-refractory prostate cancer (HRPC). Once the tumor has achieved a castration-refractory metastatic stage, treatment options are limited, and the average survival time of these patients is relatively short [10]. Furthermore, HRPC patients are often elderly or very elderly men with significant co-morbidities. Therefore, a short course of radiotherapy would be a desirable option for these patients. However, prostate cancer usually requires comparably high radiation doses, which are generally difficult to administer within very few days when using conventional radiotherapy. Moreover, in case of large treatment volumes, radiotherapy can be associated with significant toxicities, particularly in the rectum and the urinary bladder [11-14].

With modern high-precision radiotherapy techniques such as volumetric modulated arc therapy (VMAT), stereotactic body radiation therapy (SBRT) and stereotactic radiosurgery (SRS), better sparing of the organs at risk and administration of higher radiation doses in a short time can be achieved. A recent review suggested that SBRT and SRS are effective for metastatic prostate cancer [15]. Further improvement of the treatment results may be achieved by using specific diagnostic imaging procedures for treatment planning such as advanced magnetic resonance imaging (MRI) with diffusion weighted imaging (DWI) [16-20]. When using DWI, quantitative measures such as the apparent diffusion coefficient (ADC) were shown to be the most important modality for predicting tumor location and local tumor recurrences larger than $0.4 \mathrm{ml}$ [18-20]. Thus, change of the ADC can be used as a surrogate marker of response [21-25].

The present feasibility study was conducted to investigate a new short-course radiotherapy regimen for patients with metastatic HRPC presenting with a dominant debilitating symptom, for example pain, urinary retention, chronic rectal obstruction or bleeding. The number of metastases is not limited and could be one lesion (if symptomatic), oligo-metastatic disease (up to 5 lesions) or multiple metastases (more than 5 lesions). This new approach will use MRI to verify correspondence between the progressive HRPC lesion (DSL = the dominant symptomatic lesion) and the patient's dominant symptom. DWI will be used to identify both the lesion and the most aggressive part of the lesion. The DSL can be any symptomatic metastatic lesion. It will receive $4 \times 5$ Gy of highprecision radiotherapy, and the most aggressive part of the lesion $4 \times 7$ Gy using a simultaneous integrated boost (SIB) technique. Based on the results of this study, a prospective randomized phase II/III dose escalation study will be designed. Fractions of 7 Gy were recently used for hypofractionated image-guied radiotherapy for oligometastatic prostate cancer [26]. Since the Danish standard regimen for palliative treatment is short-course radiotherapy woth four fractions $(4 \times 5 \mathrm{~Gy})$, four times 7 Gy was selected for the most aggressive part of the DSL in the present study.

\section{Endpoints of the study \\ Primary endpoint}

- Feasibility, i.e. proportion of study participants who complete radiotherapy with $\geq 90 \%$ of the prescribed dose

\section{Secondary endpoints}

- Dominant symptom score (VAS)

- Progression-free survival (symptom control)

- Overall survival

- Acute toxicity (RTOG/CTCAE v.4.03)

- Quality of life (EORTC-QLQ-C30)

- Change in ADC from baseline to end of treatment

- Change in ADC from baseline to 6 months following treatment

\section{Study design}

This is an international, multi-center single arm prospective feasibility study.

\section{Inclusion criteria}

- Patients with hormone refractory biopsy proven HRPC

- Presenting with a dominant debilitating symptom

- Expected median survival of at least 3 months [5]

- Focal irradiation of lesion is feasible

- Systemic therapy according to guidelines

- Age $\geq 18$ years

- Legal capacity, patient understands the nature, significance and consequences of the study.

- Written informed consent 


\section{Exclusion criteria}

- Relevant comorbidity (with limitations to administer radiotherapy according to protocol)

- Prior radiotherapy which results in limitations to administer radiotherapy according to protocol

- No large metal implants in vicinity of lesion

- Department dose constraints for normal tissue can't be met

- Large bony lesions with extensive osseous destruction (e.g. vertebral body)

- Patients symptoms do not correlate with MRI findings

\section{Treatment}

\section{Systemic therapy}

Systemic therapy will be administered according to department standard guidelines. Systemic therapy may be chemotherapy, anti-hormonal therapy, targeted therapies, bisphosphonates or a combination. The type of therapy will be documented. Interruptions of systemic therapy, delays or changes of drugs due to radiotherapy should be avoided.

\section{Radiotherapy}

Preferred technique is hypo-fractionation with 4 fractions of high-precision radiotherapy (SBRT, SRS or VMAT). The dominant symptomatic lesion will receive $4 \times 5$ Gy of high-precision radiotherapy, and the most aggressive part of the lesion $4 \times 7$ Gy using a simultaneous integrated boost (SIB) technique. Daily IGRT is mandatory for hypo-fractionated regimens. For the treatment of thoracic lesions and metastases in the upper abdomen, techniques for motion compensation (e.g. breath-hold or gating techniques) should be used, if available. For specific situations (e.g. spinal metastases with involvement of the spinal canal or cord compression or metastases with infiltration of hollow organs), lower total doses and lower doses per fraction may be required for adherence to dose constraints and avoidance of radiation-induced complications such as perforation. In such situations, moderate hypo-fractionation with more than 4 fractions and doses per fraction $<5 \mathrm{~Gy}$ is allowed. The total dose should be equivalent to at least $50 \mathrm{~Gy}$ of conventional fractionation.

A radiologist will review the MRI scans to validate correspondence between the lesion on MRI and the patient's dominant symptom. In case of more than one lesion, decision on priority of target volumes will be made by the radiation oncologist based on clinical expertise. An ADC map will be calculated for the lesion using the DWI sequences.

The dominant symptomatic lesion (GTV1) is drawn manually by a radiologist and a radiation oncologist using the information from T2w-MRI and computed tomography
(CT) scans [27]. The most aggressive part of the dominant lesion (GTV2) is defined by using the ADC map. An auxiliary volume is created including only voxels in the GTV1 that presents with ADC values below $1200 \times 10^{-6} \mathrm{~mm}^{2} / \mathrm{s}$. This is necessary, because the GTV1 drawn by the clinicians may contain benign tissue with relatively large ADC values. The median ADC value for the auxiliary volume is then calculated. The most aggressive part of the tumor is then defined as voxels with an ADC value below this median ADC value. Notice that GTV2 need not to be one coherent volume, but may consist of several sub-volumes. In this study, the clinical target volume (CTV) is the same as the GTV (visible macroscopic tumor). The safety margin for the planning target volume (PTV) should be according to the department standard, and depending on the radiation technique used. The margin for the PTV2 for CTV2 may be chosen to be smaller than for PTV1as this volume is enclosed in the CTV1, which receives a dose of $4 \times 5$ Gy already. For the treatment of lesions moving according to breathing (e.g. in the upper abdomen), treatment techniques with motion compensation (e.g. breath-hold or gating) should be used.

\section{Assessments}

Patients are scheduled for follow-up visits at 1, 3, 6 months after end of radiotherapy. Additional MRI (T2w and DWI) will be performed at 1 and 6 months after end of radiotherapy or in case of progression of the dominant symptom. A complete staging according to current guidelines with adequate imaging is required. The available data should give sufficient information about:

- Number of visible metastatic lesions

- location of each lesion (e.g. lung metastasis in left lower lobe, osseous metastases in the sacrum)

- Size of each lesion

\section{Baseline examination}

A baseline diagnostic MRI corresponding to dominant symptom is required, and the MRI-scans must be coregistered to imaging necessary for radiotherapy planning CT. CT is allowed for judgement of the stability of bone metastases in addition to MRI. However, MRI (diffusion weighted) is required to identify the most aggressive part of DSL, and T2w MRI is needed to coregister the diffusion weighted MRI to the planning CT.

Baseline examination includes:

- Review of medical history inclusive previous anticancer treatment

- Review of clinical routine blood and radiology results

- Review of concomitant medication, analgesia, opioids, current systemic anti-cancer treatment 
- Tumor staging, grading, classification, localizations, sizes

- Physical examination (incl. Height, weight)

- Performance status (ECOG)

- Baseline dominant symptom (VAS)

- Baseline acute toxicity RTOG/CTCAE v.4.03)

- Quality of life (EORTC-QLQ C30)

\section{End of radiotherapy}

- Review of concomitant medication, analgesia, opioids, current systemic anti-cancer treatment

- Review of clinical routine blood results and radiology

- Dominant symptom score (VAS)

- Acute toxicity (RTOG/CTCAE v4.03)

- Performance status (ECOG)

- Assessment of AEs/SAEs

- Radiotherapy techniques used

- Volumes, fractions, doses applied, dose constraint compliance for all organs at risk

- Cumulative doses to target volumes, any boosts

\section{Follow-up visits after radiotherapy}

Follow-up visits will be performed at 1, 3 and 6 months after the end of radiation treatment including the following items:

- Review of concomitant medication, analgesia, opioids, current systemic anti-cancer treatment

- Review of clinical routine blood and radiology results

- Dominant symptom score (VAS)

- Acute toxicity (RTOG/CTCAE v4.03)

- Quality of life (EORTC-QLQ C30) (at 6 months only)

- Performance status (ECOG)

- Assessment of AEs/SAEs

\section{Diagnostic procedures during the follow-up period}

Protocol specific follow-up MRI scans are required for patient visit at one and six months after end of radiotherapy or at progression of the dominant symptom. Remission status evaluated at 6 month based on available clinical data. Follow-up MRI scans will be co-registered to the baseline MRI scans. Comparative volume and ADC statistics will be calculated as surrogate markers of response to radiotherapy.

\section{Sample size calculation}

The objective of this study is to investigate the feasibility of applying hypo-fractionated radiotherapy to treat a dominant symptomatic lesion in patients with HRPC. The hypothesis is that at least $90 \%$ of the recruited patients complete combined systemic and local treatment with at least $90 \%$ of scheduled radiation dose administered. We use a two-sided one-sample proportion score test to compare the proportion of feasible cases to a reference value of $90 \%$. We want to detect any difference with statistical significance of $5 \%$ and a power of $80 \%$ for not overlooking a true proportion non-feasible of $70 \%$. The necessary sample size is estimated to 24 cases. Drop-out may happen either due to exclusion, or because patients withdraw their consent for participation. For patients with poor prognosis a realistic dropout rate is estimated to be $30 \%$, which implicated that 34 patients must be included in the study to reach significance.

\section{Discussion}

Patients with metastatic PC have a better survival prognosis when compared to patients with metastases from several other solid tumors and may live for several months or even years $[28,29]$. Thus, a considerable number of these patients may live long enough to experience treatment-related late toxicities and a recurrence of the treated lesions. Many of these patients receive radiotherapy, which needs to consider several aspects including symptom control, ideally for the patients' remaining lifetime, and its feasibility [6-9]. Since PC requires higher radiation doses than many other solid tumors, the goal of delivering a high and safe dose often may not be possible with conventional RT. This problem may be solved with modern highprecision RT techniques such as SBRT, SRS and VMAT that generally allow a better sparing of the surrounding organs at risk and/or an escalation of the dose to the metastatic lesions [20,30]. Moreover, since metastatic PC is an incurable situation, the overall treatment time of radiotherapy should be kept as short as possible. Therefore, hypo-fractionated high-precision RT with very few fractions appears a reasonable option. In a palliative situation like metastatic PC, control of debilitating symptoms is very important, and often the most important goal for the patients $[6,7,9]$. Thus, one may consider focusing the treatment on the symptomatic lesions in order to avoid unnecessary toxicity by treating very large volumes and/or many sites. Since high-precision radiotherapy techniques are both resources and time consuming, the patients to be included in this study must have an expected survival prognosis of at least 3 months.

Therefore, the present study protocol has been developed that combines different aspects of radiotherapy for metastatic PC, namely high-precision RT with very few fractions focusing on the dominant symptomatic lesion. In order to deliver a high biologically effective dose allowing for long-term symptom control without increasing the risk of treatment-related toxicity, an additional SIB is administered to the most aggressive part of the dominant lesion. The present study investigates the feasibility of the novel approach, as well as response, 
symptom control, overall survival, acute toxicity and quality of life.

If this new radiotherapy regimen proves to be feasible, a prospective randomized phase II/III dose escalation study will be designed to contribute to the improvement of the outcomes of palliative radiotherapy of symptomatic metastatic HRPC in terms of better long-term symptom control and less radiation-related toxicity.

\section{Abbreviations}

ADC: apparent diffusion coefficient; AE: adverse event; CT: computed tomography; CTCAE: Common Terminology Criteria for Adverse Events; CTV: clinical target volume; DSL: dominant symptomatic lesion; DW : diffusion weighted imaging; ECOG: Eastern Cooperative Oncology Group; EORTC: European Organisation for Research and Treatment of Cancer; GTV: gross tumor volume; HRPC: hormone-refractory prostate cancer; IGRT: image-guided radiotherapy; MRI: magnetic resonance imaging; PC: prostate cancer; PTV: planning target volume; QLQ: quality of life questionnaire; RT: radiotherapy; RTOG: Radiation Therapy Oncology Group; SAE: serious adverse event; SBRT: stereotactic body radiation therapy; SIB: simultaneous integrated boost; SRS: stereotactic radiosurgery; VAS: visual analogue scale; VMAT: volumetric modulated arc therapy

\section{Acknowledgements}

The study is part of the INTERREG-project InnoCan. The authors wish to thank all colleagues and project partners working within the InnoCan project for their excellent collaboration.

\section{Funding}

The study is part of the INTERREG-project InnoCan, which is funded by the European Union (reference: Innoc 11-1.0-15). The funding body has no role in the design of the study, in collection, analysis and interpretation of the data and in writing of the manuscript.

\section{Availability of data and materials}

The study has been registered and details of the study will be available at clinicaltrials.gov (identifier: NCT03658434).

\section{Related articles}

This is the first publication related to the PRADO study.

\section{Authors' contributions}

J.C., D.R., C.D., C.S., J.D. and N.H.H. participated in the generation of the study protocol of the PRADO study. J.H.C., D.R. and N.H.H. drafted the manuscript, which has been reviewed by the other authors. The final version of the manuscript has been approved by the authors.

\section{Ethics approval and consent to participate}

The study has been approved by the ethics committee of the Zealand University Hospital, Naestved, Denmark (reference number: SJ-671). In addition, the study concept has been approved by the expert panel of DEGRO (German Association of Radiation Oncology). The study is conducted in accordance with the principles laid out in the Declaration of Helsinki and in accordance with the principles of Good Clinical Practice (ICH-GCP E6). Patients are included after giving written informed consent.

\section{Consent for publication}

Not applicable.

\section{Competing interests}

D.R. is an associate editor for BMC Cancer. Otherwise, the authors declare that they have no competing interest related to the study presented here.

\section{Publisher's Note}

Springer Nature remains neutral with regard to jurisdictional claims in published maps and institutional affiliations.

\section{Author details}

'Department of Oncology and Palliative Units, Zealand University Hospital, Naestved, Denmark. ${ }^{2}$ Department of Radiation Oncology, University of Lübeck, Lübeck, Germany. ${ }^{3}$ Department of Radiation Oncology,

Christian-Albrechts University Kiel, Kiel, Germany.

Received: 23 October 2018 Accepted: 2 January 2019

Published online: 10 January 2019

\section{References}

1. Bubendorf L, Schopfer A, Wagner U, Sauter G, Moch H, Willi N, Gasser TC, Mihatsch MJ. Metastatic patterns of prostate cancer: an autopsy study of 1,589 patients. Hum Pathol. 2000;31:578-83

2. Fossa SD, Dearnaley DP, Law M, Gad J, Newling DW, Tveter K. Prognostic factors in hormone-resistant progressing cancer of the prostate. Ann Oncol. 1992;3:361-6.

3. Otnes $B$, Harvei S, Fossa SD. The burden of prostate cancer from diagnosis until death. Br J Urol. 1995:76:587-94.

4. Zagars GK, Sands ME, Pollack A, von Eschenbach AC. Early androgen ablation for stage D1 (N1 to N3, M0) prostate cancer: prognostic variables and outcome. J Urol. 1994;151:1330-3.

5. Halabi S, Lin CY, Kelly WK, Fizazi KS, Moul JW, Kaplan EB, Morris MJ, Small EJ. Updated prognostic model for predicting overall survival in first-line chemotherapy for patients with metastatic castration-resistant prostate cancer. J Clin Oncol. 2014;32:671-7.

6. Cameron MG, Kersten C, Vistad I, van Helvoirt R, Weyde K, Undseth C, Mjaaland I, Skovlund E, Fosså SD, Guren MG. Palliative pelvic radiotherapy for symptomatic incurable prostate cancer - a prospective multicenter study. Radiother Oncol. 2015;115:314-20.

7. Din OS, Thanvi N, Ferguson CJ, Kirkbride P. Palliative prostate radiotherapy for symptomatic advanced prostate cancer. Radiother Oncol. 2009;93:192-6.

8. Gogna NK, Baxi S, Hickey B, Baumann K, Burmeister E, Holt T. Split-course, high-dose palliative pelvic radiotherapy for locally progressive hormonerefractory prostate cancer. Int J Radiat Oncol Biol Phys. 2012;83:e205-11.

9. Cameron MG, Kersten C, Guren MG, Fossa SD, Vistad I. Palliative pelvic radiotherapy of symptomatic incurable prostate cancer - a systematic review. Radiother Oncol. 2014;110:55-60.

10. James ND, Bloomfield D, Luscombe C. The changing pattern of management for hormone-refractory, metastatic prostate cancer. Prostate Cancer Prostatic Dis. 2006;:221-9.

11. Budaus L, Bolla M, Bossi A, Cozzarini C, Crook J, Widmark A, Wiegel T. Functional outcomes and complications following radiation therapy for prostate cancer: a critical analysis of the literature. Eur Urol. 2012;61:112-27.

12. Landoni V, Fiorino C, Cozzarini C, Sanguineti G, Valdagni R, Rancati T. Predicting toxicity in radiotherapy for prostate cancer. Phys Med. 2016; 32:521-32.

13. Forman JD, Keole S, Bolton S, Tekyi-Mensah S. Association of prostate size with urinary morbidity following mixed conformal neutron and photon irradiation. Int J Radiat Oncol Biol Phys. 1999;45:871-5.

14. Pinkawa M, Fischedick K, Asadpour B, Gagel B, Piroth MD, Nussen S, Eble MJ. Toxicity profile with a large prostate volume after external beam radiotherapy for localized prostate cancer. Int J Radiat Oncol Biol Phys. 2008;70:83-9.

15. Conde Moreno AJ, Ferrer Albiach C, Muelas Soria R, Gonzalez Vidal V, Garcia Gomez R, Albert Antequera M. Oligometastases in prostate cancer: restaging stage IV cancers and new radiotherapy options. Radiat Oncol. 2014;9:258.

16. Dulaney CR, Osula DO, Yang ES, Rais-Bahrami S. Prostate radiotherapy in the era of advanced imaging and precision medicine. Prostate Cancer. 2016; 2016:4897515.

17. Oberlin DT, Casalino DD, Miller FH, Meeks JJ. Dramatic increase in the utilization of multiparametric magnetic resonance imaging for detection and management of prostate cancer. Abdom Radiol (NY). 2017:42:1255-8.

18. Sun Y, Reynolds H, Wraith D, Williams S, Finnegan ME, Mitchell C, Murphy D, Ebert MA, Haworth A. Predicting prostate tumour location from multiparametric MRI using Gaussian kernel support vector machines: a preliminary study. Australas Phys Eng Sci Med. 2017:40:39-49.

19. Afaq A, Koh DM, Padhani A, van As N, Sohaib SA. Clinical utility of diffusionweighted magnetic resonance imaging in prostate cancer. BJU Int. 2011; 108:1716-22. 
20. Anwar M, Weinberg V, Seymour Z, Hsu IJ, Roach M 3rd, Gottschalk AR. Outcomes of hypofractionated stereotactic body radiotherapy boost for intermediate and high-risk prostate cancer. Radiat Oncol. 2016;11:8.

21. Decker G, Murtz P, Gieseke J, Traber F, Block W, Sprinkart AM, Leitzen C, Buchstab T, Lütter C, Schüller H, Schild HH, Willinek WA. Intensity-modulated radiotherapy of the prostate: dynamic $A D C$ monitoring by DWI at 3.0 T. Radiother Oncol. 2014;113:115-20.

22. Liu L, Wu N, Ouyang H, Dai JR, Wang WH. Diffusion-weighted MRI in early assessment of tumour response to radiotherapy in high-risk prostate cancer. Br J Radiol. 2014;87:20140359.

23. Blackledge MD, Collins DJ, Tunariu N, Orton MR, Padhani AR, Leach MO, Koh DM. Assessment of treatment response by total tumor volume and global apparent diffusion coefficient using diffusion-weighted MRI in patients with metastatic bone disease: a feasibility study. PLoS One. 2014;9:e91779.

24. Padhani AR, Makris A, Gall P, Collins DJ, Tunariu N, de Bono JS. Therapy monitoring of skeletal metastases with whole-body diffusion MRI. J Magn Reson Imagin. 2014;39:1049-78

25. Perez-Lopez R, Mateo J, Mossop H, Blackledge MD, Collins DJ, Rata M, Morgan VA, Macdonald A, Sandhu S, Lorente D, Rescigno P, Zafeiriou Z, Bianchini D, Porta N, Hall E, Leach MO, de Bono JS, Koh DM, Tunariu N. Diffusion-weighted imaging as a treatment response biomarker for evaluating bone metastases in prostate Cancer: a pilot study. Radiology. 2017;283:168-77.

26. Baumann R, Koncz M, Luetzen U, Krause F, Dunst J. Oligometastases in prostate cancer : metabolic response in follow-up PSMA-PET-CTs after hypofractionated IGRT. Strahlenther Onkol. 2018;194:318-24.

27. Morgan VA, Riches SF, Giles S, Dearnaley D, deSouza NM. Diffusion-weighted MRI for locally recurrent prostate cancer after external beam radiotherapy. AJR Am J Roentgenol. 2012;198:596-602.

28. Siegel RL, Miller KD, Jemal A. Cancer statistics, 2017. CA Cancer J Clin. 2017; 67:7-30.

29. Rades D, Douglas S, Veninga T, Stalpers LJ, Hoskin PJ, Bajrovic A, Adamietz IA, Basic H, Dunst J, Schild SE. Validation and simplification of a score predicting survival in patients irradiated for metastatic spinal cord compression. Cancer. 2010;116:3670-3.

30. Ishii K, Ogino R, Hosokawa Y, Fujioka C, Okada W, Nakahara R, Kawamorita R, Tada T, Hayashi Y, Nakajima T. Whole-pelvic volumetric-modulated arc therapy for high-risk prostate cancer: treatment planning and acute toxicity. J Radiat Res. 2015;56:141-50.

Ready to submit your research? Choose BMC and benefit from:

- fast, convenient online submission

- thorough peer review by experienced researchers in your field

- rapid publication on acceptance

- support for research data, including large and complex data types

- gold Open Access which fosters wider collaboration and increased citations

- maximum visibility for your research: over $100 \mathrm{M}$ website views per year

At $\mathrm{BMC}$, research is always in progress.

Learn more biomedcentral.com/submissions 